\title{
A simple optical fiber device for quantitative fluorescence microscopy of single living cells
}

\author{
Marja van Graft, Bernard Oosterhuis, Kees O. van der Werf, Bart G. de Grooth \\ and Jan Greve \\ Department of Applied Physics, Division of Applied Optics, University of Twente, P.O. Box 217, 7500 AE Enschede, Netherlands
}

(Received 20 July 1992, revised received 4 September 1992, accepted 30 September 1992)

\begin{abstract}
A simple and relatively inexpensive system is described for obtaining quantitative fluorescence measurements on single living cells loaded with a fluorescent probe to study cell physiological processes. The light emitted from the fluorescent cells is captured by and transported through an optical fiber. After passage through appropriate filters the light is measured using a photomultiplier tube. The optical fiber is mounted in one of the microscope outlets. Signals derived from the photomultiplier are converted to voltage, amplified, and displayed on a recorder. In the excitation pathway a shutter control unit is mounted. With this control unit the period that the excitation pathway is 'opened' and 'closed' can be adjusted, to reduce cell damage and/or bleaching of the probe. This option allows time-lapse recording of experiments up to $1 \mathrm{~h}$. We have used this set-up with a single and dual emission fluorescent probe to determine intracellular calcium concentrations and $\mathrm{pH}$, respectively. In Fluo-3-loaded $\mathrm{K} 562$ target cells bound to natural killer cells, a temporary rise in $\left[\mathrm{Ca}^{2+}\right]_{i}$ was accompanied by bleb formation. The simple construction of this set-up is interchangeable between different types of fluorescence microscopes and can easily be combined with other microscopy techniques, e.g., patch clamp.
\end{abstract}

Key words: Quantitative fluorescence microscopy; Natural killer cell; K562 cell; Fluo-3; Seminaphthorhodafluor 1; Patch clamp

\section{Introduction}

In the last decade the use of fluorochromes in cell characterization and cell physiology studies has become a very valuable tool. Many new fluorescent probes with a great diversity of applica-

Correspondence to: M. van Graft, Department of Applied Physics, University of Twente, P.O. Box 217, 7500 AE Enschede, Netherlands. Tel.: +31-53-893157; Fax: +31-53309549.

Abbreviations: CM, culture medium; FCS, fetal calf serum; NK, natural killer; PMT, photomultiplier; SNARF, seminaphthorhodafluor; SWP, short wave pass. tions have been developed (for an overview see: Lansing Taylor and Wang, 1989; Tsien, 1989). For characterization studies, as in, e.g., immunophenotyping of cells or localization of cell organelles, qualitative detection of the fluorescence signal is, in most cases, sufficient. However, in order to study time-dependent physiological processes in single living cells, a quantitative detection system for continuous measurement is required. Nowadays many microscopic fluorimetry systems have been developed using photomultipliers or photodiodes for time-resolved quantitative fluorescence measurements on single cells (Nobiling and Bührle, 1989; Vergara et al., 1991). 
Using advanced cameras and computer systems, time-and space-resolved quantitative fluorescence measurements can be performed (Connor, 1986; Hiraoka et al., 1987; Vergara et al., 1991). With these systems detailed information can be obtained in relation to the quantity and localization of a probe inside single living cells. However, the systems available have several disadvantages: they are very expensive, the measurements performed are discontinuous instead of continuous and, furthermore, they are not easy to apply to different microscopes. This led us to develop a simple device for quantitative fluorescence microscopy. The following conditions had to be fulfilled: (1) the device had to be sensitive; (2) it had to be composed of inexpensive components; (3) it had to be compatible with different types of fluorescence microscope. In the present paper the design and properties of the device are described as well as its application to the study of intracellular calcium transients in single K562 target cells during cytotoxic interactions with a natural killer cell. Dual emission fluorescence measurement was demonstrated using seminaphthorhodafluor 1 (SNARF-1) loaded K562 cells.

\section{Materials and methods}

\section{A device for quantitative fluorescence microscopy}

A schematic representation of the device is shown in Fig. 1. A fiber (the refractive indices $(n)$ of core and cladding being 1.460 and 1.404 , respectively; N.A. 0.40, Cunz, Frankfurt/M, Germany) with a $600 \mu \mathrm{m}$ core diameter and about 1 $\mathrm{m}$ long was mounted in one of the ocular tubes of a fluorescence microscope. The entrance surface of the fiber was polished and it was mounted 160 $\mathrm{mm}$ behind the objective in the calculated image field where the incoming beam of light has low divergence and is captured entirely by the fiber. The diameter of the fiber permitted recording of the image of a single cell (for calculations: see results section). Loss of emitted light due to leaky waves can be excluded. At the opposite end, the transported light passes a diverging lens $(f=20$ $\mathrm{mm}$, Spindler and Hoyer, Göttingen, Germany), followed by appropriate filter combinations: filter 1 (Fluo-3 and F-18 emission) consists of OG515 and KV520 filters and an short wave pass (SWP) filter of $550 \mathrm{~nm}$; filter 2 (SNARF-1 with an emission of $580 \mathrm{~nm}$ ) transmits a band between $530-600$ $\mathrm{nm}$, and consists of KV520 and OG530 filters and an SWP filter of $600 \mathrm{~nm}$; filter 3 (SNARF-1 645 $\mathrm{nm}$ emission) is transmitting between 600-650 $\mathrm{nm}$, and consists of an OG610 and an SWP 650 $\mathrm{nm}$ filter (all filters were obtained from Oriel, Stratford, CT). The light then passes a converging lens $(f=60 \mathrm{~mm})$ and finally reaches the cathode of a photomultiplier (PMT, type R928 Hamamatsu, Bridgewater, NJ). The PMT signal is converted to voltage and after amplification it is recorded. At the excitation side the light $(100 \mathrm{~W}$ mercury arc with a DC stabilized power supply) passes through the standard cubic dichroic mirror unit resulting in either blue or green excitation light (Olympus IMT2-DMB or IMT2-DMG, respectively). To reduce adverse effects of excitation light - bleaching of the fluorochrome or damage to living cells - the amount of excitation

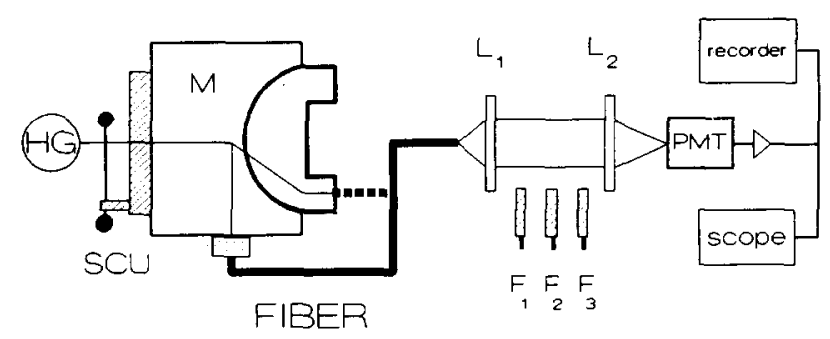

Fig. 1. Schematic representation of the optical fiber device developed for quantitative fluorescence measurements using an inverted microscope. M: microscope; HG: mercury arc lamp house; SCU: shutter control unit; FIBER: optical fiber mounted on the side outlet (solid line) or on an ocular outlet (broken line); $L_{1}$ and $L_{2}$ : lenses with foci of 20 and $60 \mathrm{~mm}$, respectively; $F_{1}, F_{2}$ and $F_{3}$ : emission filters (for details see text); PMT: photomultiplier. 
light is reduced as much as possible. Therefore, in the fluorescence illuminator the aperture and the field diaphragm are set to a minimal opening. For long term experiments, the standard excitation light shutter of the vertical fluorescence illuminator is used. The shutter is attached to a linear stage (travel length $50 \mathrm{~mm}$ ) and driven by a 4 W DC motor (type 2140934, Maxon, Interelectric, Sachseln, Switzerland). The motor speed is controlled by a linear servo control module (type MMC QR 030 024-02LD00A, Maxon). With this control unit the excitation path can either be closed or opened continuously (manual mode) or with a defined repetition time and duty cycle (auto mode) with a range of a total time of 0-99 s and an open time of $0-9 \mathrm{~s}$. In the results shown here a neutral density filter is placed in the 'opened' position reducing the excitation light to $25 \%$. The device described here was used on an Olympus IMT-2 inverted microscope. In our setup the fiber was placed in the side outlet. This had two advantages. Firstly, both eye pieces could be used for observation of the specimen and secondly, a better yield was obtained since $80 \%$ of the emitted light was detected instead of only $50 \%$ if one of the eye pieces was used.

\section{Cell cultures}

The natural killer (NK) cell clone was cultured as described by Bolhuis et al. (1984). Once a week the NK cells were harvested and maintained in 24 well dishes (Costar, Cambridge, MA) at a concentration of $5 \times 10^{5}$ cells $/ \mathrm{ml}$. As culture medium (CM) RPMI 1640 without phenol red (Seramed, Berlin, Germany) was used. CM was buffered with $10 \mathrm{mM}$ Hepes and $2 \mathrm{mM}$ bicarbonate and supplemented with $10 \%$ heat-inactivated fetal calf serum (FCS, Gibco, Gaithersburg, MD), $2 \mathrm{mM}$ L-glutamine, $100 \mathrm{U} / \mathrm{ml}$ penicillin $\mathrm{G}, 100$ $\mu \mathrm{g} / \mathrm{ml}$ streptomycin (Sigma Chem. Comp., St. Louis, MO). For culturing NK cells $25 \mathrm{U} / \mathrm{ml}$ human recombinant IL-2 (Cetus, Amsterdam, Netherlands, and a kind gift from Dr. R.L.H. Bolhuis) was added to CM. K562 cells were cultured in CM buffered only with $2.4 \mathrm{mM}$ bicarbonate. The cultures were kept at $37^{\circ} \mathrm{C}$ in an incubator with a humidified atmosphere and $5 \% \mathrm{CO}_{2}$. During the cytotoxic experiments all cells were kept in CM supplemented with calcium at a final concentration of $2 \mathrm{mM} \mathrm{Ca}^{2+}(\mathrm{CM}+)$.

Loading procedures for Fluo-3, SNARF-1, and F-18 Fluo-3 was used as $\mathrm{Ca}^{2+}$ indicator (Kao et al., 1989; Minta et al., 1989), and SNARF-1 was used to indicate the intracellular pH (Whitaker et al., 1988). Both Fluo-3 and SNARF-1 were readily loaded into the cells as acetoxymethylesters (Fluo-3-AM and SNARF-1-AM, Molecular Probes, Eugene, OR). For loading a $10 \mu \mathrm{M}$ Fluo3-AM working solution was prepared in $\mathrm{CM}$ that contained $0.5 \%(\mathrm{v} / \mathrm{v})$ of a solution of pluronic F-127 (Molecular Probes) in dry dimethylsulfoxide $(25 \% \mathrm{w} / \mathrm{w})$. The cells $\left(10^{7} / \mathrm{ml}\right)$ were mixed $1: 1$ with the working solution and kept at $37^{\circ} \mathrm{C}$ in an incubator. During incubation the cells were gently agitated. After $60 \mathrm{~min}$ the cells were washed three times with cold $\mathrm{CM}$ and stored in a refrigerator until use. For loading of SNARF-1 the same procedure was used except that K562 cells were loaded in CM without FCS. Nigericin ( $2 \mu \mathrm{g} / \mathrm{ml}$, Molecular Probes) was used as a proton ionophore to obtain a calibration curve. Octadecylaminefluorescein isothiocyanate (F-18), a stable membrane probe (Koolwijk et al., 1988), was used as described previously by Radoševic et al. (1990).

\section{Microscopy}

Experiments were carried out in a temperature-controlled micro-incubator (Ince et al., 1983). Temperature was controlled to either $25^{\circ} \mathrm{C}$ or $37^{\circ} \mathrm{C}$, via the top ring of the dish holder, resulting in stable temperature control of the sample. Cells were placed in a teflon culture dish (Ince et al., 1985). The bottom of the culture dish consisted of a coverslip coated with $0.001 \%$ poly-L-lysine (Sigma Chem. Comp.) which allowed cells to adhere. The cell suspension was covered with Klearol $(0.6-1 \mathrm{ml}$, Witco chemical, New York, NY) to reduce evaporation. Fluorescence measurements were carried out with a planapochromat $\times 40$ UV dry objective with an N.A. of 0.85 and coverslip correction between $0.11-0.23 \mathrm{~mm}$ (Olympus 31804). Cell morphology was observed with a planachromat $\times 40$ phase-contrast objective (Olympus 31612). 


\section{Cytotoxic interactions}

To study cytotoxic interactions, NK cells and K562 cells were brought together in the teflon culture dish. K562 cells were attached to the poly-L-lysine-coated coverslips in about $15 \mathrm{~min}$, whereas some of the NK cells maintained their mobility, and their ability to form conjugates with K562 cells. Active conjugates were characterized by a change in shape of the NK cell (such as flattening) due to strong adherence to the K562 cell. In such a conjugate, after 5-15 min, spherical structures, so-called blebs, appeared from the membrane of the K562 cell due to the cytotoxic action of the NK cell. Conjugates, consisting of an NK cell with a round appearance, and a K562 cell with no blebs were considered to be non-active conjugates. To study the $\left[\mathrm{Ca}^{2+}\right]_{i}$ in NK cells and K562 cells during cytotoxic interactions one of the cell types was loaded with Fluo-3. The conjugates formed consisted of one Fluo-3-loaded and one unloaded cell. Changes in the Fluo-3 signal were recorded over time in cells that were part of active or non-active conjugates. Single cells were used as controls.

\section{Results}

\section{Specification of the optical fiber device}

A sensitive device for quantitative fluorescence measurements was constructed using an optical fiber and a photomultiplier (PMT). In this set up noise was signal-limited. The fiber was positioned in the center of the insert. The center was marked by a cross in the photo frame reticle. It was established that the position of the fiber coincided with the center of the object plane. The field diameter in which an object was measured with this fiber, was determined using fluorescent microbeads with a diameter of $0.93 \mu \mathrm{m}$. A $\mu \mathrm{m}$ scale was placed in the object plane and the focussed microbead was moved over certain distances. Using a $\times 40$ objective a sharp decrease of the fluorescence signal was observed if the bead was moved more than $5 \mu \mathrm{m}$ from the center (Fig. 2). This indicated that the effective field diameter in the object plane was about $10-12 \mu \mathrm{m}$. With the $\times 40$ objective, a cell having a diameter of 10 $\mu \mathrm{m}$ produced an image of $400 \mu \mathrm{m}$ diameter

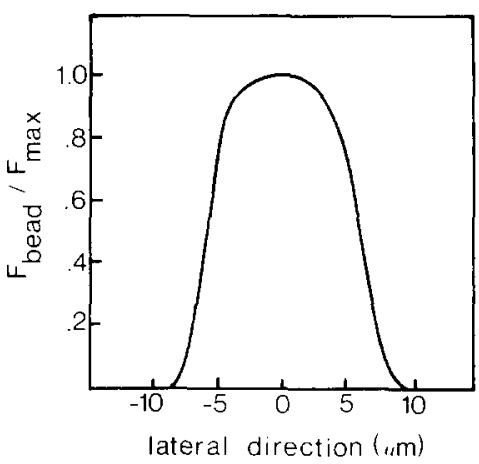

Fig. 2. Relationship between the fluorescence signal $\left(F_{\text {bead }} / F_{\max }\right)$ of a fluorescent microbead $(\emptyset, 0.93 \mu \mathrm{m})$, and the lateral distance to the center of the object plane. The fluorescence signal was normalized to $F_{\max }$, the maximum signal of the microbead centered in the object plane. Positioning of the bead in the lateral direction was performed using an ocular with $\mu \mathrm{m}$ scale. A $40 \times$ objective was used.

which fitted totally in the fiber used. 'Depth of focus' was determined by making a scan of a centered microbead $(0.93 \mu \mathrm{m})$ in the $z$ axis (Fig. 3 ). With the $\times 40$ objective a value of $18 \mu \mathrm{m}$ was obtained. This high value was due to the low divergence of the imaging beam. Consequently, the effective volume from which the fluorescence signal was detected was calculated to have a maximum of about $1400 \mu \mathrm{m}^{3}$. A spherical cell with a radius of $5 \mu \mathrm{m}$ has a volume of $523 \mu \mathrm{m}^{3}$ ( $\sim 37 \%$ of the effective volume). This means that

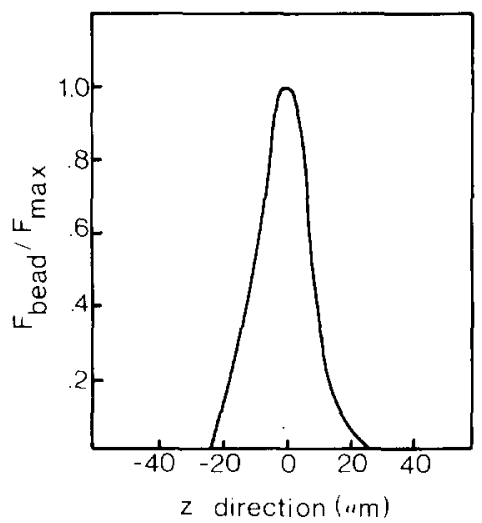

Fig. 3. Relation of the fluorescence signal $\left(F_{\text {bead }} / F_{\text {max }}\right)$ of a microbead $(\emptyset, 0.93 \mu \mathrm{m})$ centered in the object plane, and the distance in the $z$ axis. The fluorescence signal was normalized to the maximum signal of the centered microbead $\left(F_{\max }\right)$. A depth profile of the fluorescence signal of the bead was obtained by displacement of the objective $(40 \times)$ in the $z$ axis. 
measuring the fluorescence signal of a cell with a diameter of $10 \mu \mathrm{m}$, one has to account for a contribution of the surrounding culture medium of about $60 \%$ of the effective volume.

\section{Time-dependent changes of fluorochrome signals in unstimulated cells}

During continuous detection of fluorochrome light emission, time-dependent reduction of the fluorescence signal can be expected due to bleaching, leakage, or a combination of both phenomena. Due to the small area of measurement, reduction of the signal in our set up may also be caused by movement of a centered cell out of the object field or out of focus, for example due to vibration caused by the shutter, pipetting, or cell mobility. To study these possibilities immobile NK cells loaded with F-18 were used. From previous studies (Radoševic et al., 1990) we know that leakage of this dye could be neglected. A typical result is shown in Fig. 4. When cells were illuminated continuously, a decrease of the fluorescence signal was observed (Fig. $4 a$ ). The rate of this decrease was reduced when illumination of the cells was interrupted at a certain frequency by a dark period (Fig. $4 b$ ). From these results it was concluded that signal reduction was caused by bleaching. As no steep decrease of the signal was observed the cell stayed in the object field. Thus, movement of the cell or of the microscope due to vibration of the shutter can be excluded, which

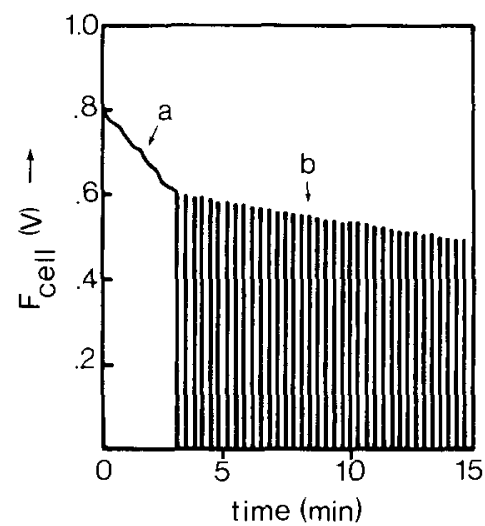

Fig. 4. Time-lapse recordings of the fluorescence signal of an F-18 loaded NK cell. $a$ : shutter continuously open; $b$ : shutter open for $5 \mathrm{~s}$ and closed for $15 \mathrm{~s}$.

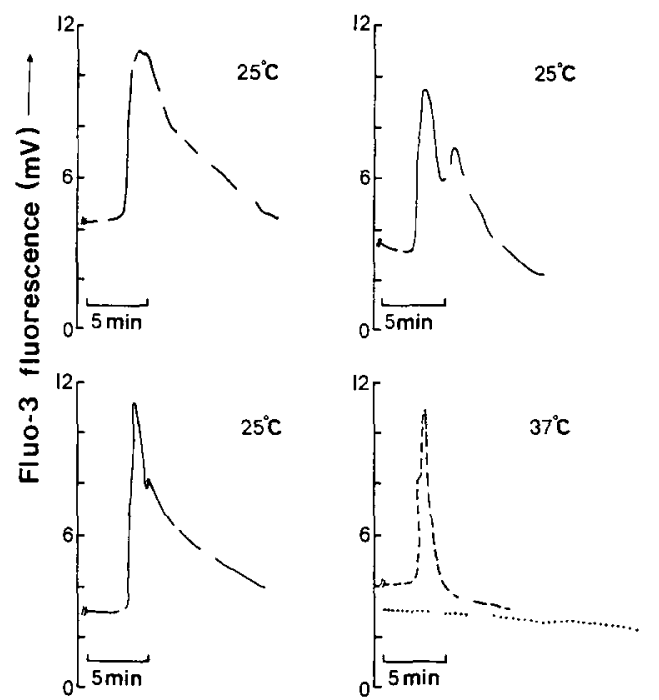

Fig. 5. Changes of the fluorescence signals derived from Fluo-3 loaded K562 cells in conjugate with NK cells. Conjugates were monitored for several minutes. Here, only parts of the recordings are shown which present the increase in the Fluo-3 signal. Parts in which the signal was stable are omitted. Of four different K562 cells conjugated to an active NK cell, the redrawn recordings are shown: three at $25^{\circ} \mathrm{C} \mathrm{(-)}$ and one at $37^{\circ} \mathrm{C} \mathrm{(-} \mathrm{-} \mathrm{Also} \mathrm{the} \mathrm{signal} \mathrm{from} \mathrm{a} \mathrm{K562}$ cell conjugated to an inactive $\mathrm{NK}$ cell $(\cdots \cdots)$ is shown. The measurements were interrupted by phase-contrast observations. The shutter was open continuously.

implies that the optical fiber attachment to the microscope and the microscope stage are stable. Pipetting of additives, when carefully performed, had no effect on the fluorescence signal.

\section{Changes of the fluorescence signal in stimulated cells}

During the cytotoxic interaction of NK cells and K562 cells intracellular events were studied using fluorescent probes. Interactions between the cells leading to formation of conjugates were followed using phase-contrast microscopy. When an active conjugate was found, the loaded cell of that particular conjugate was centered in the cross of the photo frame reticle. Phase-contrast illumination was turned off and detection of the fluorescence signal commenced. Morphological changes occurring in the cells during fluorescence detection could be observed by interruption of the measurement. In Fig. 5 parts of the Fluo-3 
signals derived from conjugated K562 cells are shown. These measurements on target cells forming part of active conjugates were started before bleb formation of the K562 cell was observed. No changes in Fluo-3 fluorescence signals were then detected (Fig. 5, dotted line). In some cases conjugated $\mathrm{K} 562$ cells showed a rapid increase in the Fluo-3 fluorescence signal (Fig. 5, solid lines). The increase was temperature independent, whereas the decrease of the signal was faster at $37^{\circ} \mathrm{C}$ (Fig. 5, broken line). During the descending phase at $25^{\circ} \mathrm{C}$ it was possible to make fluorescence measurements and alternately look at the conjugates by phase-contrast. In these experiments we observed that an increase in the Fluo-3 signal was accompanied by bleb formation of the conjugated K562 cell. After the Fluo-3 fluorescence had returned to normal values, bleb formation continued. It was thus concluded that in so-called active conjugates during the initial phase of bleb formation in the target cell a temporary rise of the $\left[\mathrm{Ca}^{2+}\right]_{i}$ level occurred. In a target cell conjugated with a cytotoxic $\mathrm{T}$ lymphocyte, the increase of the $\left[\mathrm{Ca}^{2+}\right]_{i}$ is more permanent (Poenie et al., 1987) in contrast with our result. These authors did not report bleb formation. In suspensions of $\mathrm{K} 562$ cells mixed with NK cells the $\left[\mathrm{Ca}^{2+}\right]_{i}$ in the target cell population was also reported to be increased (McConkey et al., 1990).

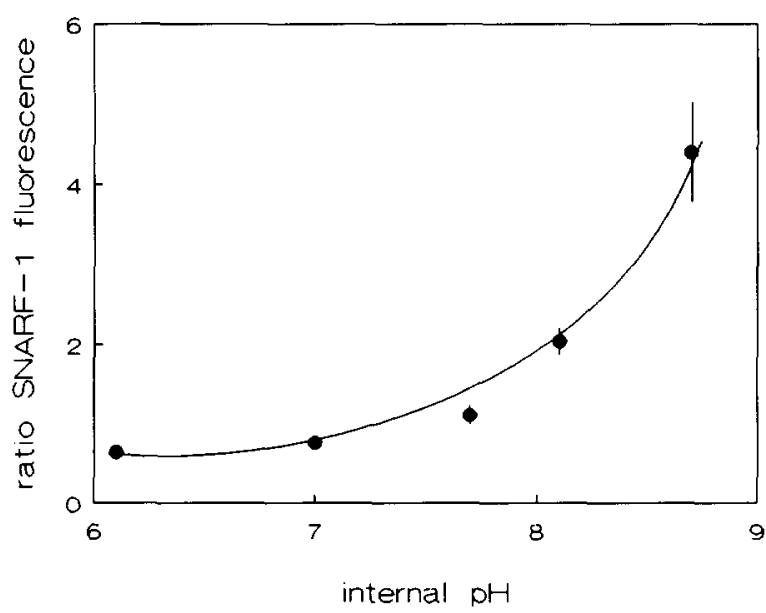

Fig. 6. Calibration curve of SNARF-1 loaded K562 cells. The intracellular $\mathrm{pH}$ of the cells was clamped to the indicated $\mathrm{pH}$ values using nigericin containing potassium buffers. $n=4$ for each $\mathrm{pH}$ value.
Since in the latter study bulk measurements were performed the transient character of the calcium rise could have been masked since the cytotoxic interactions do not occur synchronously.

\section{pH measurements on single cells using dual emis-} sion detection

To demonstrate ratio measurements, SNARF1-loaded K562 cells were used. The calibration curve was established as previously described (Rabinovitch and June, 1990). K562 cells were suspended in nigericin containing buffers of known $\mathrm{pH}$. Fluorescence signals were recorded using alternately filters 2 and 3 to detect the 580 $\mathrm{nm}$ and $645 \mathrm{~nm}$ signal, respectively. The ratio of the fluorescence signals as a function of the intracellular $\mathrm{pH}$ is shown in Fig. 6. This result is in agreement with those obtained by Van Erp et al. (1991) and Martinez-Zaguilan et al. (1991).

\section{Discussion}

In this paper a device is described for quantitative fluorescence measurements by microscopy on single living cells during cell physiological processes. Due to its simple construction this lowbudget device can be built in almost any laboratory environment and costs about $10-20 \%$ of the commercially available photomultiplier systems. Application of the device to different types of microscope is easily accomplished by transferring the fiber insert. A motorized shutter control unit mounted at the back of the microscope body in the excitation light path permits quantitative time-lapse measurements to be performed for prolonged periods with minimum bleaching and cell damage due to the light source. We have used this device to demonstrate its convenience in different applications that are used in the current research field of fluorescent ion probes. It can be used for single emission detection studies as we have shown with the calcium sensitive probe Fluo-3. As ratio probes are preferred for many quantitative applications, we also performed studies using the pH-indicator SNARF-1. It was shown that dual emission detection can be carried out easily and, if desired, ratio measurements can be optimized using a dichroic mirror in 
the light emitted from the fiber and a second PMT. After dividing the two signals immediate registration of the desired fluorescence ratio signal would be possible. It is concluded that the simple low-budget device described can be a useful tool for performing quantitative fluorescence studies on living cells with standard fluorescence microscopes and can be used as an additional technique in, for example, patch-clamp studies.

\section{Acknowledgements}

This work was supported by a grant of the Netherlands Organization for Scientific Research (N.W.O.) through the Foundation of Biophysics. The authors express their thanks to Prof. Dr. D.L. Ypey for helpful discussions.

\section{References}

Bolhuis, R.L.H., Van de Griend, R.J. and Ronteltap, C.P.M. (1984) Clonal expansion of human B-73.1-positive natural killer cells or large granular lymphocytes exerting strong antibody-dependent and antibody-independent cytotoxicity and occasionally lectin-dependent cytotoxicity. Natl. Immun. Cell Growth Regul. 3, 61.

Connor, J.A. (1986) Digital imaging of free calcium changes and of spatial gradients in growing processes in single, mammalian central nervous system cells. Proc. Natl. Acad. Sci. USA 83, 6179.

Hiraoka, Y., Sedat, J.W. and Agard, D.A. (1987) The use of charge-coupled device for quantitative optical microscopy of biological structures. Science 238, 36 .

Ince, C., Ypey, D.L., Diesselhoff-Den Dulk, M.M.C., Visser, J.A.M., De Vos, A. and Van Furth, R. (1983) Micro- $\mathrm{CO}_{2}$ incubator for use on a microscope. J. Immunol. Methods $60,269$.

Ince, C., Van Dissel, J.T. and Diesselhoff, M.M.C. (1985) A teflon culture dish for high-magnification microscopy and measurements in single cells. Pflügers Arch. 403, 240.

Kao, J.P.Y., Harootunian, A.T. and Tsien, R.Y. (1989) Photo- chemically generated cytosolic calcium pulses and their detection by Fluo-3. J. Biol. Chem. 264, 8179.

Koolwijk, P., Rozemuller, E., Stad, R.K., De Lau, W.B.M. and Bast, B.J.E.G. (1988) Enrichment and selection of hybridomas by Percoll density gradient centrifugation and fluorescence-activated cell sorting. Hybridoma 7, 217.

Lansing Taylor, D. and Wang, Y.-L. (Eds.) (1989) Methods in Cell Biology, Vol. 29. Academic Press, San Diego, CA.

Martinez-Zaguilan, R., Martinez, G.M., Lattanzio, F. and Gillies, R.J. (1991) Simultaneous measurement of intracellular $\mathrm{pH}$ and $\mathrm{Ca}^{2+}$ using the fluorescence of SNARF-1 and Fura-2. Am. J. Physiol. 260 (Cell Physiol. 29), C297.

McConkey, D.J., Chow, S.C., Orrenius, S. and Jondal, M. (1990) NK cell-induced cytotoxicity is dependent on a $\mathrm{Ca}^{2+}$ increase in the target. FASEB J. 4, 2662.

Minta, A., Kao, J.P.Y. and Tsien, R.Y. (1989) Fluorescent indicators for cytosolic calcium based on rhodamine and fluorescein chromophores. J. Biol. Chem. 264, 8171.

Nobiling, R. and Bührle, C.P. (1989) A microscopic fluorimeter using multiple-wavelength excitation for ultrasensitive single-cell emission spectrometry. J. Microsc. 156, 149.

Poenie, M., Tsien, R.Y. and Schmitt-Verhulst, A.-M. (1987) Sequential activation and lethal hit measured by $\left[\mathrm{Ca}^{2+}\right]_{i}$ in individual cytolytic $\mathrm{T}$ cells and targets. EMBO J. 6, 2223.

Rabinovitch, P.S. and June, C.H. (1990) In: M.G. Ormerod (Ed.), Intracellular ionized calcium, membrane potential, and $\mathrm{pH}$. Flow cytometry: a practical approach. Oxford University Press, New York, p. 161.

Radoševic, K., Garritsen, H.S.P., Van Graft, M., De Grooth, B.G. and Greve, J. (1990) A simple and sensitive flow cytometric assay for the determination of the cytotoxic activity of human natural killer cells. J. Immunol. Methods $135,81$.

Tsien, R.Y. (1989) In: D. Lansing Taylor and Y.-L. Wang (Eds.), Fluorescent Indicators of Ion Concentrations, Vol. 30, Methods in Cell Biology. Academic Press, San Diego, CA, p. 127.

Van Erp, P.E.J., Jansen, M.J.J.M., De Jongh, G.J., Boezeman, J.B.M. and Schalkwijk, J. (1991) Ratiometric measurement of intracellular $\mathrm{pH}$ in cultured human keratinocytes using carboxy-SNARF-1 and flow cytometry. Cytometry 12, 127.

Vergara, J., DiFranco, M., Compagnon, D. and Suarez-Isla, B.A. (1991) Imaging of calcium transients in skeletal muscle fibers. Biophys. J. 59, 12.

Whitaker, J.E., Haugland, R.P. and Prendergast, F.G. (1988) Seminaphtofluoresceins and -rhodafluors: dual fluorescence $\mathrm{pH}$ indicators. Biophys. J. 53, 197a. 